\title{
Novel Formulas for Displacements and Stresses of Thick Anisotropic Rectangular Plate
}

\author{
Ibearugbulem $\mathrm{OM}^{1}$, Ezeh $\mathrm{JC}^{2}$, Ozioko $\mathrm{HO}^{3 *}$, and Anya $\mathrm{UC}^{4}$ \\ ${ }^{1,2,4}$ Department of Civil Engineering Federal University of Technology, Owerri, Imo State, Nigeria \\ ${ }^{3}$ Department of Civil Engineering, Michael Okpara University of Agriculture, Umudike, Abia State, Nigeria
}

DOI: $10.36348 /$ sjce.2020.v04i09.002

| Received: 15.10 .2020 | Accepted: 25.10.2020 | Published: 05.11.2020

*Corresponding author: Hyginus Obinna Ozioko

\section{Abstract}

This work concentrated on the analysis of thick anisotropic rectangular plate through exact approach using third order shear deformation theory. Refined plate theory assumptions were relied upon to formulate the total potential energy functional. Displacement field, kinematic relations, constitutive relations and stress displacement relations were also obtained from the assumptions. Kinematic relations and Stress-displacement relations were substituted into the universal strain energy equation to formulate the strain energy equation. Total potential energy functional for the analysis of thick anisotropic rectangular plate was obtained by adding the external work and strain energy equation together. The total potential energy functional was differentiated with respect to the out plane deflection (w), shear deformation rotation in $\mathrm{x}$ direction $\left(\phi_{x}\right)$ and shear deformation rotation in y direction $\left(\phi_{y}\right)$. This yielded the governing equation and two compatibility equations of thick anisotropic rectangular plate. Third order polynomial shear deformation function which was derived by Ibearugbulem et al. was relied upon to obtain the displacement functions. From these displacement functions, the unique displacement functions for the SSSS plate boundary condition were determined. Also the stiffness coefficients were calculated for the SSSS plate boundary condition. The formulas for calculating the coefficients of the displacements were combined with elastic equations to determine the novel formulas which were used in calculating for displacements ( $\mathrm{u}, \mathrm{v}$ and $\mathrm{w})$ and stresses $\left(\sigma_{\mathrm{RR}}, \sigma_{\mathrm{QQ}}, \tau_{\mathrm{RQ}}, \tau_{\mathrm{RS}}\right.$ and $\left.\tau_{\mathrm{QS}}\right)$ at various angle fiber orientation $\left(0^{\circ}, 15^{\circ}, 30^{\circ}\right.$, $45^{\circ}, 60^{\circ}, 75^{\circ}$ and $\left.90^{\circ}\right)$ and various span to thickness ratio, $\alpha(5,10,20,30,40,50,60,70,80,90$ and 100). These formula were used to analyze typical anisotropic rectangular thick plates. The results obtained were shown on Tables 1 , 2, 3, 4 and 5. These numerical results obtained showed some level of agreement with previous works by other scholars. Hence the developed method is recommended for analyzing thick rectangular anisotropic plates.

Keywords: Energy; Anisotropic; Thick Plate; Displacement; Compatibility and Governing Equation.

Copyright () 2020 The Author(s): This is an open-access article distributed under the terms of the Creative Commons Attribution 4.0 International License (CC BY-NC 4.0) which permits unrestricted use, distribution, and reproduction in any medium for non-commercial use provided the original author and source are credited.

\section{INTRODUCTION}

Any material consisting of two or more components with different properties and distinct boundaries between the components can be referred to as a composite material [1]. Anisotropic plate is a composite material with two or more components that possesses different properties. Advances in the application of thick and anisotropic plates over the past few decades have stirrup a revival of the concerned of researchers to formulate appropriate numerical methods and tools to bode the structural behavior of anisotropic plates. Hence, a precise, simple and cost effective numerical method that is suitable to be applied to anisotropic composite thick plate is of exceptional interest and therefore, various anisotropic composite plate theories and numerical method have already been formulated to predict the behavior of anisotropic composite plates. Among these methods is the polynomial shear deformation theory that is well meant for the effective analysis of anisotropic composite plate structures.

Anisotropic composite plate is one of the most important structural materials used in engineering industries such as marine, astronautic, aeronautics, electronics, computer, etc. This is as a result of high strength to weight and stiffness to weight ratios of anisotropic composite materials, which makes them conforming to an ultimate standard of perfection in weight sensitive structures. The dynamic distinguishing quality of the anisotropic composite plate have a great act of alluring engineers and material scientist. 
Various solutions and models established in the literature for analysis of anisotropic plate did not derive specific formulas for calculation of displacement and stresses. Yang et al analyzed for the bending, free vibration and buckling analyses of anisotropic layered micro-plates based on a new size dependent model [2]. Lisboa and Marczak applied adomian decomposition method to anisotropic thick plate in bending [3]. Ghugal and Shimpi reviewed a refined shear deformation theories of isotropic and anisotropic laminated plates [4]. Nelson and Lorch worked on a refined theory for laminated orthotropic plates [5]. Phan and Reddy applied a higher order shear deformation theory to obtain the stability and vibration of isotropic, orthotropic and laminated plates [6].

One common observation is that most of these works are based mainly on trigonometric and assumed displacement functions. These works could not determine specific formulas for calculation of displacements and stresses which has been a major obstacle in easy understanding of thick anisotropic plate analysis. It is believed that deriving specific formulas for displacements and stresses will enhance the understandability of the solution and as well guide the engineers to easy usability of the solution in thick anisotropic rectangular plate. Another observation is that earlier works on anisotropic thick plates had relied on assumed displacement functions (which are mainly trigonometric) and could not determine specific formulas for calculation of displacements and stresses of thick anisotropic rectangular plate. Those works always have to follow a rigorous processes/method each time to calculate for displacements and stresses. This long processes can be very difficult and tiring in thick anisotropic plate analysis. Hence, the need to derive formulas which can easily determine displacements and stresses is very necessary in thick anisotropic plate analysis. To cover this gap in anisotropic thick plate analysis is the primary motivation of the present study.

Based on the above observations, it can be concluded that there is need for the development of formulas for displacements and stresses of thick rectangular anisotropic plate subjected under bending loading which can accurately calculate for in-plane displacements, out-plane displacement, in-plane stresses and out-plane stresses. With above points, the main objective of the present study is to develop novel formulas for bending responses of anisotropic rectangular plate.

Hence, this paper gives the results for displacements and stresses of thick anisotropic and as well isotropic plates of SSSS boundary condition.

\section{Theoritical formulation}

To obtain the novel formulas for the analysis of thick anisotropic plate, third order shear deformation theory and Ritz energy method were adopted. The method is described in this research work as follows;

\section{Displacement field}

The refined plate theory (RPT) in-plane displacements, $\mathrm{u}$ and $\mathrm{v}$ are defined mathematically as presented:

$$
\begin{aligned}
& u=u_{c}+u_{s} \\
& v=v_{c}+v_{s}
\end{aligned}
$$

The non-dimensional forms of the orthogonal axes are defined as: $\mathrm{R}=\mathrm{x} / \mathrm{a} ; \mathrm{Q}=\mathrm{y} / \mathrm{b} ; \mathrm{S}=\mathrm{z} / \mathrm{t}$. aspect ratio, denoted as $\beta$ is defined as $\beta=b / a$.

The classical part of the in-plane displacements $\mathrm{u}_{\mathrm{c}}$ and $\mathrm{v}_{\mathrm{c}}$ are defined as follows:

$$
\begin{aligned}
& u_{c}=-z \theta_{c x}=-z \frac{d w}{d x}=-\frac{S t}{a} \frac{d w}{d R} \\
& v_{c}=-z \theta_{c y}=-z \frac{d w}{d y}=-\frac{S t}{b} \frac{d w}{d R}=-\frac{S t}{\beta a} \frac{d w}{d Q}
\end{aligned}
$$

Where $\mathrm{w}$, is the out-plane displacement. Transverse displacements $\mathrm{u}_{\mathrm{s}}$ and $\mathrm{v}_{\mathrm{s}}$ are defined as:

$$
\begin{aligned}
& u_{s}=F(z) \theta_{s x} \\
& v_{s}=F(z) \theta_{s y}
\end{aligned}
$$

Where: $F(z)$ is the third order shear deformation model defined as:

$$
F(z)=z-\frac{4}{3} \cdot \frac{z^{3}}{t^{2}}=z\left(1-\frac{4}{3}\left[\frac{z}{t}\right]^{2}\right)
$$

The non-dimensional form of the model is:

That is:

$$
F=F(s)=t\left(S-\frac{4}{3} S^{3}\right)
$$

$$
F=t H
$$

Where: 


$$
H=S-\frac{4}{3} S^{3}
$$

Adding Equations 3 and 5 gives:

$$
u=-\frac{S t}{a} \frac{d w}{d R}+F(z) \cdot \emptyset_{x}
$$

Similarly, adding Equations 4 and 6 gives:

$$
v=-\frac{S t}{\beta a} \frac{d w}{d Q}+F(z) . \emptyset_{y}
$$

Substituting Equation 7c into Equations $8 \mathrm{a}$ and $8 \mathrm{~b}$ gives:

$$
\begin{aligned}
& u=\frac{t}{a}\left[-S \frac{\partial w}{\partial R}+H a . \emptyset_{x}\right] \\
& v=\frac{t}{a \beta}\left[-S \frac{\partial w}{\partial Q}+\beta H a . \emptyset_{y}\right]
\end{aligned}
$$

\section{Strain - displacement relations (kinematic relations)}

The strain - displacement relations equations are:

$$
\begin{aligned}
& \varepsilon_{R}=\frac{\partial u}{\partial x}=\frac{\partial u}{a \partial R}=\frac{t}{a^{2}}\left[-S \frac{\partial^{2} w}{\partial R^{2}}+H a \cdot \frac{\partial \phi_{x}}{\partial R}\right] \\
& \varepsilon_{Q}=\frac{\partial v}{\partial y}=\frac{\partial v}{a \beta \partial Q}=\frac{t}{\beta^{2} a^{2}}\left[-S \frac{\partial^{2} w}{\partial Q^{2}}+H a \beta \cdot \frac{\partial \phi_{y}}{\partial Q}\right] \\
& \gamma_{R Q}=\varepsilon_{R Q}+\varepsilon_{Q R}=\frac{\partial u}{\partial y}+\frac{\partial v}{\partial x}=\frac{t}{\beta a^{2}}\left[-S \frac{\partial^{2} w}{\partial R \partial Q}+H a \cdot \frac{\partial \phi_{x}}{\partial Q}\right]+\frac{t}{\beta a^{2}}\left[-S \frac{\partial^{2} w}{\partial R \partial Q}+H \beta a \cdot \frac{\partial \phi_{y}}{\partial R}\right] . \text { That is: } \\
& \gamma_{R Q}=\frac{t}{\beta a^{2}}\left[-2 S \frac{\partial^{2} w}{\partial R \partial Q}+H a \cdot\left(\frac{\partial \phi_{x}}{\partial Q}+\beta \cdot \frac{\partial \phi_{y}}{\partial R}\right)\right] \\
& \gamma_{R S}=\varepsilon_{R S}+\varepsilon_{S R}=\frac{\partial u}{\partial z}+\frac{\partial w}{\partial x}=\frac{1}{a}\left[-\frac{\partial w}{\partial R}+a \frac{\partial H}{\partial S} \cdot \emptyset_{x}\right]+\frac{1}{a} \frac{\partial w}{\partial R} . \text { That is: } \\
& \gamma_{R S}=\frac{\partial H}{\partial S} \cdot \emptyset_{x} \\
& \gamma_{Q S}=\frac{\partial H}{\partial S} \cdot \emptyset_{y}=\frac{\partial v}{\partial z}+\frac{\partial w}{\partial y}=\frac{1}{\beta a}\left[-\frac{\partial w}{\partial Q}+\beta a \frac{\partial H}{\partial S} \cdot \emptyset_{y}\right]+\frac{1}{\beta a} \cdot \frac{\partial w}{\partial Q} \text {. That is: } \\
& \gamma_{Q S}=\frac{\partial H}{\partial S} \cdot \emptyset_{y}
\end{aligned}
$$

\section{Constitutive relations (Stress - Strain Relations)}

$$
\left[\begin{array}{c}
\sigma_{R} \\
\sigma_{Q} \\
\tau_{R Q} \\
\tau_{R S} \\
\tau_{Q S}
\end{array}\right]=\frac{E_{0}}{1-\mu_{12} \mu_{21}}\left[\begin{array}{ccccc}
B_{11} & B_{12} & B_{13} & 0 & 0 \\
B_{21} & B_{22} & B_{23} & 0 & 0 \\
B_{31} & B_{32} & B_{33} & 0 & 0 \\
0 & 0 & 0 & B_{44} & 0 \\
0 & 0 & 0 & 0 & B_{55}
\end{array}\right]\left[\begin{array}{c}
\varepsilon_{R} \\
\varepsilon_{Q} \\
\gamma_{R Q} \\
\gamma_{R S} \\
\gamma_{Q S}
\end{array}\right]
$$

Where:

$\mathrm{E}_{0}$ is the reference Elastic modulus. It can be $\mathrm{E}_{1}$ or $\mathrm{E}_{2} ; m=\operatorname{Cos} \theta ; n=\operatorname{Sin} \theta$

$$
\begin{aligned}
& B_{11}=m^{4} d_{11}+2 m^{2} n^{2}\left(d_{12}+2 d_{33}\right)+n^{4} d_{22} \\
& B_{12}=d_{12}\left(n^{4}+m^{4}\right)+m^{2} n^{2}\left(d_{11}+d_{22}-4 d_{33}\right) \\
& B_{13}=m^{3} n\left(d_{11}-d_{12}-2 d_{33}\right)+m n^{3}\left(d_{12}-d_{22}+2 d_{33}\right) \\
& B_{22}=n^{4} d_{11}+2 m^{2} n^{2}\left(d_{12}+2 d_{33}\right)+m^{4} d_{22} \\
& B_{23}=m n^{3} d_{11}-m^{3} n d_{22}+\left(m^{3} n-m n^{3}\right)\left(d_{12}+2 d_{33}\right) \\
& B_{33}=m^{2} n^{2}\left(d_{11}-2 d_{12}+d_{22}-2 d_{33}\right)+d_{33}\left(m^{4}+n^{4}\right) \\
& B_{44}=d_{44} ; B_{55}=d_{55} ; B_{21}=B_{12} ; B_{31}=B_{13} ; B_{32}=B_{23} \\
& d_{11}=E_{1} / E_{0} \\
& d_{12}=E_{2} \cdot \mu_{12} / E_{0} \\
& d_{21}=E_{1} \cdot \mu_{21} / E_{0} \\
& d_{22}=E_{22} / E_{0} \\
& d_{33}=G_{12}\left(1-\mu_{12} \mu_{21}\right) / E_{0} \\
& d_{44}=G_{13}\left(1-\mu_{12} \mu_{21}\right) / E_{0}
\end{aligned}
$$




$$
d_{55}=G_{23}\left(1-\mu_{12} \mu_{21}\right) / E_{0}
$$

Substituting Equations 9 to 13 into Equation 14 gives each stress component as:

$$
\begin{aligned}
& \sigma_{\mathrm{R}}=\frac{\mathrm{E}_{0} \mathrm{t}}{\left[1-\mu_{12} \mu_{21}\right] a^{2}} \cdot\left(\mathrm{B}_{11} \cdot\left[-S \frac{\partial^{2} w}{\partial R^{2}}+H a \cdot \frac{\partial \phi_{x}}{\partial R}\right]+\frac{\mathrm{B}_{12}}{\beta^{2}} \cdot\left[-S \frac{\partial^{2} w}{\partial Q^{2}}+H a \beta \cdot \frac{\partial \phi_{y}}{\partial Q}\right]\right. \\
& \left.+\frac{B_{13}}{\beta} \cdot\left[-2 S \frac{\partial^{2} w}{\partial R \partial Q}+H a \cdot\left(\frac{\partial \phi_{x}}{\partial Q}+\beta \cdot \frac{\partial \phi_{y}}{\partial R}\right)\right]\right) \\
& \sigma_{\mathrm{Q}}=\frac{\mathrm{E}_{0} \mathrm{t}}{\left[1-\mu_{12} \mu_{21}\right] a^{2}} \cdot\left(\mathrm{B}_{21} \cdot\left[-S \frac{\partial^{2} w}{\partial R^{2}}+H a \cdot \frac{\partial \phi_{x}}{\partial R}\right]+\frac{\mathrm{B}_{22}}{\beta^{2}} \cdot\left[-S \frac{\partial^{2} w}{\partial Q^{2}}+H a \beta \cdot \frac{\partial \phi_{y}}{\partial Q}\right]\right. \\
& \left.+\frac{B_{23}}{\beta} \cdot\left[-2 S \frac{\partial^{2} w}{\partial R \partial Q}+H a \cdot\left(\frac{\partial \phi_{x}}{\partial Q}+\beta \cdot \frac{\partial \phi_{y}}{\partial R}\right)\right]\right) \\
& \tau_{\mathrm{RQ}}=\frac{\mathrm{E}_{0} \mathrm{t}}{\left[1-\mu_{12} \mu_{21}\right] a^{2}} \cdot\left(\mathrm{B}_{31} \cdot\left[-S \frac{\partial^{2} w}{\partial R^{2}}+H a \cdot \frac{\partial \phi_{x}}{\partial R}\right]+\frac{\mathrm{B}_{32}}{\beta^{2}} \cdot\left[-S \frac{\partial^{2} w}{\partial Q^{2}}+H a \beta \cdot \frac{\partial \phi_{y}}{\partial Q}\right]\right. \\
& \left.+\frac{\mathrm{B}_{33}}{\beta} \cdot\left[-2 S \frac{\partial^{2} w}{\partial R \partial Q}+H a \cdot\left(\frac{\partial \phi_{x}}{\partial Q}+\beta \cdot \frac{\partial \phi_{y}}{\partial R}\right)\right]\right) \\
& \tau_{\mathrm{RS}}=\frac{\mathrm{E}_{0}}{1-\mu_{12} \mu_{21}} \cdot \mathrm{B}_{44} \cdot\left[\frac{\partial H}{\partial S}\right] \cdot \emptyset_{x}=\frac{\mathrm{E}_{0} \mathrm{t}}{\left[1-\mu_{12} \mu_{21}\right] a^{2}} \cdot \mathrm{B}_{44} \cdot\left[\frac{a^{2}}{\mathrm{t}} \cdot \frac{\partial H}{\partial S}\right] \cdot \emptyset_{x} \\
& \tau_{\mathrm{QS}}=\frac{\mathrm{E}_{0}}{1-\mu_{12} \mu_{21}} \cdot \mathrm{B}_{55} \cdot\left[\frac{\partial H}{\partial S}\right] \cdot \emptyset_{y}=\frac{\mathrm{E}_{0} \mathrm{t}}{\left[1-\mu_{12} \mu_{21}\right] a^{2}} \cdot \mathrm{B}_{55} \cdot\left[\frac{a^{2}}{\mathrm{t}} \cdot \frac{\partial H}{\partial S}\right] \cdot \emptyset_{y}
\end{aligned}
$$

\section{Total potential energy functional}

The total potential energy functional is given as:

$$
\begin{gathered}
\Pi=\frac{\mathrm{abt}}{2} \int_{0}^{1} \int_{0}^{1} \int_{-0.5}^{0.5}\left(\sigma_{\mathrm{R}} \varepsilon_{\mathrm{R}}+\sigma_{\mathrm{R}} \varepsilon_{\mathrm{R}}+\tau_{\mathrm{RQ}} \gamma_{\mathrm{RQ}}+\tau_{\mathrm{RS}} \gamma_{\mathrm{RS}}+\tau_{\mathrm{QS}} \gamma_{\mathrm{QS}}\right) \mathrm{dR} \mathrm{dQ} \mathrm{dS} \\
- \text { qab } \int_{0}^{1} \int_{0}^{1} \mathrm{wdRdQ}
\end{gathered}
$$

Substituting Equations 9 to 13 and Equations 29 to 33 into Equations 34 gives:

$$
\begin{aligned}
& \Pi=\frac{\mathrm{abD}_{0}}{2 a^{4}} \cdot \int_{0}^{1} \int_{0}^{1}\left\{\mathrm{~B}_{11} \cdot\left[\left(\frac{\partial^{2} w}{\partial R^{2}}\right)^{2}-2 g_{2} a \cdot \frac{\partial^{2} w}{\partial R^{2}} \cdot \frac{\partial \phi_{x}}{\partial R}+g_{3} a^{2} \cdot\left(\frac{\partial \phi_{x}}{\partial R}\right)^{2}\right]\right. \\
& +\frac{\mathrm{B}_{12}}{\beta^{2}} \cdot\left[2\left(\frac{\partial^{2} w}{\partial R \partial Q}\right)^{2}-g_{2} \frac{a}{\beta} \frac{\partial^{2} w}{\partial Q^{2}} \cdot \frac{\partial \phi_{y}}{\partial Q}-g_{2} a \beta^{2} \frac{\partial^{2} w}{\partial R^{2}} \cdot \frac{\partial \phi_{x}}{\partial R}-g_{2} a \cdot \frac{\partial \phi_{x}}{\partial R} \cdot \frac{\partial^{2} w}{\partial Q^{2}}-g_{2} a \beta \cdot \frac{\partial^{2} w}{\partial R^{2}} \cdot \frac{\partial \phi_{y}}{\partial Q}\right. \\
& \left.+2 g_{3} a^{2} \beta \cdot \frac{\partial \phi_{x}}{\partial R} \cdot \frac{\partial \phi_{y}}{\partial Q}\right] \\
& +\frac{B_{13}}{\beta} \cdot\left[4 \frac{\partial^{2} w}{\partial R \partial Q} \cdot \frac{\partial^{2} w}{\partial R^{2}}-2 g_{2} a \cdot\left(\frac{\partial \phi_{x}}{\partial Q}+\beta \cdot \frac{\partial \phi_{y}}{\partial R}\right) \cdot \frac{\partial^{2} w}{\partial R^{2}}-4 g_{2} a \frac{\partial^{2} w}{\partial R \partial Q} \cdot \frac{\partial \phi_{x}}{\partial R}\right. \\
& \left.+2 g_{3} a^{2} \cdot\left(\frac{\partial \phi_{x}}{\partial Q}+\beta \cdot \frac{\partial \phi_{y}}{\partial R}\right) \cdot \frac{\partial \phi_{x}}{\partial R}\right]+\frac{\mathrm{B}_{22}}{\beta^{4}} \cdot\left[\left(\frac{\partial^{2} w}{\partial Q^{2}}\right)^{2}-2 g_{2} a \beta \cdot \frac{\partial^{2} w}{\partial Q^{2}} \cdot \frac{\partial \phi_{y}}{\partial Q}+g_{3} a^{2} \beta^{2} \cdot\left(\frac{\partial \phi_{y}}{\partial Q}\right)^{2}\right] \\
& +\frac{B_{23}}{\beta^{3}} \cdot\left[4 \frac{\partial^{2} w}{\partial R \partial Q} \cdot \frac{\partial^{2} w}{\partial Q^{2}}-2 g_{2} a \cdot\left(\frac{\partial \phi_{x}}{\partial Q}+\beta \cdot \frac{\partial \phi_{y}}{\partial R}\right) \cdot \frac{\partial^{2} w}{\partial Q^{2}}-4 g_{2} a \beta \cdot \frac{\partial^{2} w}{\partial R \partial Q} \cdot \frac{\partial \phi_{y}}{\partial Q}\right. \\
& \left.+2 g_{3} a^{2} \beta \cdot\left(\frac{\partial \phi_{x}}{\partial Q}+\beta \cdot \frac{\partial \phi_{y}}{\partial R}\right) \cdot \frac{\partial \phi_{y}}{\partial Q}\right] \\
& +\frac{\mathrm{B}_{33}}{\beta^{2}} \cdot\left[4\left(\frac{\partial^{2} w}{\partial R \partial Q}\right)^{2}-2 g_{2} a \cdot\left(\frac{\partial^{2} w}{\partial R \partial Q} \cdot \frac{\partial \phi_{x}}{\partial Q}+\beta \frac{\partial^{2} w}{\partial R \partial Q} \cdot \frac{\partial \phi_{y}}{\partial R}\right)\right. \\
& \left.+g_{3} a^{2} \cdot\left(\left(\frac{\partial \phi_{x}}{\partial Q}\right)^{2}+2 \beta \frac{\partial \phi_{x}}{\partial Q} \cdot \frac{\partial \phi_{y}}{\partial R}+\beta^{2} \cdot\left(\frac{\partial \phi_{y}}{\partial R}\right)^{2}\right)\right]+a^{2} \mathrm{~B}_{44} \cdot\left(\frac{a}{t}\right)^{2} \cdot g_{4} \cdot \emptyset_{x}{ }^{2} \\
& \left.\left.+a^{2} \mathrm{~B}_{55} \cdot\left(\frac{a}{t}\right)^{2} \cdot g_{4} \cdot \emptyset_{y}{ }^{2}\right\}-2 \frac{\mathrm{q} a^{4}}{\mathrm{D}_{0}} w\right\} \mathrm{dR} \mathrm{dQ}
\end{aligned}
$$


Where:

$$
D_{0}=\frac{E_{0} t^{3}}{12\left[1-\mu_{12} \mu_{21}\right]}
$$

\section{Governing equation and compatibility equations}

Differentiating Equation 35 with respect to $\mathrm{w}, \theta_{\mathrm{x}}$ and $\theta_{\mathrm{y}}$ gives the governing equation and compatibility equations respectively.

$$
\frac{\mathrm{d} \Pi}{\mathrm{dw}}=\frac{\mathrm{d} \Pi}{\mathrm{d} \emptyset_{\mathrm{x}}}=\frac{\mathrm{d} \Pi}{\mathrm{d} \emptyset_{\mathrm{y}}}=0
$$

That is:

$$
\begin{aligned}
& \frac{\mathrm{d} \Pi}{\mathrm{d} w}=\int_{0}^{1} \int_{0}^{1}\left\{\mathrm{~B}_{11} \cdot \frac{\partial^{4} \mathrm{w}}{\partial \mathrm{R}^{4}}+\frac{2}{\beta^{2}} \cdot \mathrm{B}_{\mathrm{xy}} \frac{\partial^{4} \mathrm{w}}{\partial \mathrm{R}^{2} \partial \mathrm{Q}^{2}}+\frac{\mathrm{B}_{22}}{\beta^{4}} \cdot \frac{\partial^{4} \mathrm{w}}{\partial \mathrm{Q}^{4}}+4 \frac{\mathrm{B}_{13}}{\beta} \cdot \frac{\partial^{4} \mathrm{w}}{\partial \mathrm{R}^{3} \partial \mathrm{Q}}+4 \frac{\mathrm{B}_{23}}{\beta^{3}} \cdot \frac{\partial^{4} \mathrm{w}}{\partial \mathrm{R} \partial \mathrm{Q}^{3}}\right. \\
& -\frac{\mathrm{g}_{2} \mathrm{a}}{2}\left[2 \mathrm{~B}_{11}+\mathrm{B}_{12}\right] \frac{\partial^{3} \phi_{\mathrm{x}}}{\partial \mathrm{R}^{3}}-\frac{\mathrm{g}_{2} \mathrm{a}}{2 \beta^{2}} \cdot \mathrm{B}_{\mathrm{xy}} \frac{\partial^{3} \phi_{\mathrm{x}}}{\partial \mathrm{R} \partial \mathrm{Q}^{2}}-3 \mathrm{~g}_{2} \mathrm{a} \cdot \frac{\mathrm{B}_{13}}{\beta} \frac{\partial^{3} \phi_{\mathrm{x}}}{\partial \mathrm{R}^{2} \partial \mathrm{Q}}-\frac{\mathrm{g}_{2} \mathrm{a}}{2 \beta^{3}}\left[\mathrm{~B}_{12}+2 \mathrm{~B}_{22}\right] \frac{\partial^{3} \phi_{\mathrm{y}}}{\partial \mathrm{Q}^{3}} \\
& \left.-\frac{g_{2} a}{2 \beta} B_{x y} \frac{\partial^{3} \phi_{y}}{\partial R^{2} \partial Q}-3 g_{2} a \cdot \frac{B_{23}}{\beta^{2}} \frac{\partial^{3} \phi_{y}}{\partial R \partial Q^{2}}-g_{2} a \cdot B_{13} \cdot \frac{\partial^{3} \phi_{y}}{\partial R^{3}}-\frac{g_{2} a}{\beta^{3}} \cdot B_{23} \cdot \frac{\partial^{3} \phi_{x}}{\partial Q^{3}}-\frac{q^{4}}{D_{0}}\right\} d R d Q \\
& =0 \quad 38 \\
& \frac{\mathrm{d} \Pi}{\mathrm{d} \emptyset_{\mathrm{x}}}=\mathrm{B}_{11} \cdot\left[-g_{2} a \cdot \frac{\partial^{3} w}{\partial R^{3}}+g_{3} a^{2} \cdot \frac{\partial^{2} \phi_{x}}{\partial R^{2}}\right]+\frac{\mathrm{B}_{12}}{2 \beta^{2}} \cdot\left[-g_{2} a \beta^{2} \frac{\partial^{3} w}{\partial R^{3}}-g_{2} a \cdot \frac{\partial^{3} w}{\partial R \partial Q^{2}}+2 g_{3} a^{2} \beta \cdot \frac{\partial^{2} \phi_{y}}{\partial R \partial Q}\right] \\
& +\frac{B_{13}}{\beta} \cdot\left[-g_{2} a \cdot \frac{\partial^{3} w}{\partial Q \partial R^{2}}-2 g_{2} a \frac{\partial^{3} w}{\partial Q \partial R^{2}}+2 g_{3} a^{2} \cdot \frac{\partial^{2} \phi_{x}}{\partial R \partial Q}+g_{3} a^{2} \cdot \beta \cdot \frac{\partial^{2} \phi_{y}}{\partial R^{2}}\right] \\
& +\frac{B_{23}}{\beta^{3}} \cdot\left[-g_{2} a \cdot \frac{\partial^{3} w}{\partial Q^{3}}+g_{3} a^{2} \beta \cdot \frac{\partial^{2} \phi_{y}}{\partial Q^{2}}\right]+\frac{B_{33}}{\beta^{2}} \cdot\left[-g_{2} a \cdot \frac{\partial^{3} w}{\partial R \partial Q^{2}}+g_{3} a^{2} \cdot \frac{\partial^{2} \phi_{x}}{\partial Q^{2}}+g_{3} a^{2} \cdot \beta \frac{\partial^{2} \phi_{y}}{\partial R \partial Q}\right] \\
& +a^{2} \mathrm{~B}_{44} \cdot\left(\frac{a}{t}\right)^{2} \cdot g_{4} \cdot \emptyset_{x}=0 \\
& 39 \\
& \frac{\mathrm{d} \Pi}{\mathrm{d} \emptyset_{\mathrm{y}}}=\frac{\mathrm{B}_{12}}{2 \beta^{2}} \cdot\left[-g_{2} \frac{a}{\beta} \frac{\partial^{3} w}{\partial Q^{3}}-g_{2} a \beta \cdot \frac{\partial^{3} w}{\partial R^{2} \partial Q}+2 g_{3} a^{2} \beta \cdot \frac{\partial^{2} \phi_{x}}{\partial R \partial Q}\right]+\frac{B_{13}}{\beta} \cdot\left[-g_{2} a \cdot \beta \cdot \frac{\partial^{3} w}{\partial R^{3}}+g_{3} a^{2} \cdot \beta \cdot \frac{\partial^{2} \phi_{x}}{\partial R^{2}}\right] \\
& +\frac{\mathrm{B}_{22}}{\beta^{4}} \cdot\left[-g_{2} a \beta \cdot \frac{\partial^{3} w}{\partial Q^{3}}+g_{3} a^{2} \beta^{2} \cdot \frac{\partial^{2} \phi_{y}}{\partial Q^{2}}\right] \\
& +\frac{B_{23}}{\beta^{3}} \cdot\left[-g_{2} a \cdot \beta \cdot \frac{\partial^{3} w}{\partial R \partial Q^{2}}-2 g_{2} a \beta \cdot \frac{\partial^{3} w}{\partial R \partial Q^{2}}+g_{3} a^{2} \beta \cdot \frac{\partial^{2} \phi_{x}}{\partial Q^{2}}+2 g_{3} a^{2} \beta^{2} \cdot \frac{\partial^{2} \phi_{y}}{\partial R \partial Q}\right] \\
& +\frac{\mathrm{B}_{33}}{\beta^{2}} \cdot\left[-g_{2} a \cdot \beta \frac{\partial^{3} w}{\partial R^{2} \partial Q}+g_{3} a^{2} \cdot \beta \frac{\partial^{2} \phi_{x}}{\partial R \partial Q}+g_{3} a^{2} \cdot \beta^{2} \cdot \frac{\partial^{2} \phi_{y}}{\partial R^{2}}\right]+a^{2} \mathrm{~B}_{55} \cdot\left(\frac{a}{t}\right)^{2} \cdot g_{4} \cdot \emptyset_{y} \\
& =0
\end{aligned}
$$

Equations 38, 39 and 40 are the governing equation of equilibrium of forces, compatibility equation of displacements in $\mathrm{x}-\mathrm{z}$ plane and compatibility equation of displacements in $\mathrm{y}-\mathrm{z}$ plane respectively.

\section{Solutions of governing equation and compatibility equations}

Solving Equations 38, 39 and 40 gives:

$$
\begin{aligned}
& w=A_{1} h \\
& w=\left(\alpha_{0}+\alpha_{1} \mathrm{R}+\alpha_{2} \mathrm{R}^{2}+\alpha_{3} \mathrm{R}^{3}+\alpha_{4} \mathrm{R}^{4}\right)\left(\lambda_{0}+\lambda_{1} \mathrm{Q}+\lambda_{2} \mathrm{Q}^{2}+\lambda_{3} \mathrm{Q}^{3}+\lambda_{4} \mathrm{Q}^{4}\right) \\
& \phi_{\mathrm{x}}=\frac{\mathrm{A}_{2}}{\mathrm{a}} \cdot \frac{\partial \mathrm{h}}{\partial \mathrm{R}} \\
& \emptyset_{\mathrm{y}}=\frac{\mathrm{A}_{3}}{\mathrm{a} \beta} \cdot \frac{\partial \mathrm{h}}{\partial \mathrm{Q}}
\end{aligned}
$$

Satisfying the boundary condition for SSSS plates gives their distinct deflection equation respectively as: $w=A_{1} h=\alpha_{3} \alpha_{4}\left(\mathrm{R}-2 \mathrm{R}^{3}+\mathrm{R}^{4}\right)\left(\mathrm{Q}-2 \mathrm{Q}^{3}+\lambda_{4} \mathrm{Q}^{4}\right) \quad\{$ for SSSS $\}$

$41 c$ 
Substituting Equations 41a, 42 and 43 into Equation 35 gives:

$$
\begin{aligned}
\Pi=\frac{\mathrm{ab}}{2 a^{4}} \cdot\left\{\left\{\mathrm{B}_{11}\right.\right. & \cdot\left[\mathrm{A}_{1}{ }^{2}-2 g_{2} \mathrm{~A}_{1} \mathrm{~A}_{2} \cdot+g_{3} \mathrm{~A}_{2}{ }^{2}\right] k_{1}+\frac{\left(\mathrm{B}_{12}+2 \mathrm{~B}_{33}\right)}{\beta^{2}} \cdot\left[2 \mathrm{~A}_{1}{ }^{2}-g_{2} \mathrm{~A}_{1} \mathrm{~A}_{2}-g_{2} \mathrm{~A}_{1} \mathrm{~A}_{3}\right] \cdot k_{2} \\
& +2 \frac{\left[\mathrm{B}_{12}+\mathrm{B}_{33}\right]}{\beta^{2}} g_{3} \mathrm{~A}_{2} \mathrm{~A}_{3} \cdot k_{2}+\frac{\mathrm{B}_{12}}{\beta^{2}} \cdot g_{2}\left[-\frac{\mathrm{A}_{1} \mathrm{~A}_{3}}{\beta^{2}} k_{3}-\mathrm{A}_{1} \mathrm{~A}_{2} \beta^{2} k_{1}\right]+\frac{\mathrm{B}_{33}}{\beta^{2}} \cdot\left[+g_{3} \mathrm{~A}_{2}{ }^{2}+g_{3} \mathrm{~A}_{3}{ }^{2}\right] k_{2} \\
& +\frac{B_{13}}{\beta} \cdot\left[4 \mathrm{~A}_{1}{ }^{2}-2 g_{2}\left(\mathrm{~A}_{1} \mathrm{~A}_{2}+\mathrm{A}_{1} \mathrm{~A}_{3}\right)-4 g_{2} \mathrm{~A}_{1} \mathrm{~A}_{2}+2 g_{3}\left(\mathrm{~A}_{2}{ }^{2}+\mathrm{A}_{2} \mathrm{~A}_{3}\right)\right] k_{4} \\
& +\frac{\mathrm{B}_{22}}{\beta^{4}} \cdot\left[\mathrm{A}_{1}{ }^{2}-2 g_{2} \mathrm{~A}_{1} \mathrm{~A}_{3}+g_{3} \mathrm{~A}_{3}{ }^{2}\right] k_{3} \\
& +\frac{B_{23}}{\beta^{3}} \cdot\left[4 \mathrm{~A}_{1}{ }^{2}-2 g_{2}\left(\mathrm{~A}_{1} \mathrm{~A}_{2}+\mathrm{A}_{1} \mathrm{~A}_{3}\right)-4 g_{2} \mathrm{~A}_{1} \mathrm{~A}_{3}+2 g_{3}\left(\mathrm{~A}_{2} \mathrm{~A}_{3}+\mathrm{A}_{3}{ }^{2}\right)\right] k_{5}+\mathrm{B}_{44} \cdot\left(\frac{a}{t}\right)^{2} \cdot g_{4} \cdot \mathrm{A}_{2}{ }^{2} k_{6} \\
& \left.\left.+\frac{\mathrm{B}_{55}}{\beta^{2}} \cdot\left(\frac{a}{t}\right)^{2} \cdot g_{4} \mathrm{~A}_{3}{ }^{2} k_{7}\right\}-2 \mathrm{~A}_{1} \frac{\mathrm{q}^{4}}{\mathrm{D}_{0}} k_{8}\right\}
\end{aligned}
$$

Note:

$\mathrm{k}_{1}=\int_{0}^{1} \int_{0}^{1}\left(\frac{\mathrm{d}^{2} \mathrm{~h}}{\mathrm{dR}^{2}}\right)^{2} \mathrm{dR} \mathrm{dQ} ; \mathrm{k}_{2}=\int_{0}^{1} \int_{0}^{1}\left(\frac{\mathrm{d}^{2} \mathrm{~h}}{\mathrm{dRdQ}}\right)^{2} \mathrm{dRdQ} ; \mathrm{k}_{3}=\int_{0}^{1} \int_{0}^{1}\left(\frac{\mathrm{d}^{2} \mathrm{~h}}{\mathrm{dQ}^{2}}\right)^{2} \mathrm{dR} \mathrm{dQ}$

$\mathrm{k}_{4}=\int_{0}^{1} \int_{0}^{1}\left(\frac{\mathrm{d}^{2} \mathrm{~h}}{\mathrm{dR}^{2}}\right)\left(\frac{\mathrm{d}^{2} \mathrm{~h}}{\mathrm{dRdQ}}\right) \mathrm{dR} \mathrm{dQ} ; \mathrm{k}_{5}=\int_{0}^{1} \int_{0}^{1}\left(\frac{\mathrm{d}^{2} \mathrm{~h}}{\mathrm{dQ}^{2}}\right)\left(\frac{\mathrm{d}^{2} \mathrm{~h}}{\mathrm{dRdQ}}\right) \mathrm{dR} \mathrm{dQ}$

$\mathrm{k}_{6}=\int_{0}^{1} \int_{0}^{1}\left(\frac{\mathrm{dh}}{\mathrm{dR}}\right)^{2} \mathrm{dR} \mathrm{dQ} ; \mathrm{k}_{7}=\int_{0}^{1} \int_{0}^{1}\left(\frac{\mathrm{dh}}{\mathrm{dQ}}\right)^{2} \mathrm{dR} \mathrm{dQ} ; \mathrm{k}_{8}=\int_{0}^{1} \int_{0}^{1} \mathrm{hdR} \mathrm{dQ}$

To obtain the quasi equations of equilibrium of forces and quasi compatibility equations, Equation 44 must be differentiated with respect to $\mathrm{A} 1, \mathrm{~A} 2$ and $\mathrm{A} 3$. That is:

$$
\begin{aligned}
& \frac{\mathrm{d} \Pi}{\mathrm{dA}_{1}}=\frac{\mathrm{d} \Pi}{\mathrm{dA}_{2}}=\frac{\mathrm{d} \Pi}{\mathrm{dA}_{3}}=0 \\
& \frac{\mathrm{d} \Pi}{\mathrm{dA}}=\mathrm{L}_{11} \mathrm{~A}_{1}-\mathrm{L}_{12} \mathrm{~A}_{2}-\mathrm{L}_{13} \mathrm{~A}_{3}-\frac{\mathrm{q} a^{4}}{\mathrm{D}_{0}} \mathrm{k}_{8}=0 \\
& \frac{\mathrm{d} \Pi}{\mathrm{dA}}=\mathrm{L}_{12} \mathrm{~A}_{1}-\mathrm{L}_{22} \mathrm{~A}_{2}-\mathrm{L}_{23} \mathrm{~A}_{3}=0 \\
& \frac{\mathrm{d} \Pi}{\mathrm{dA}}=\mathrm{L}_{13} \mathrm{~A}_{1}-\mathrm{L}_{23} \mathrm{~A}_{2}-\mathrm{L}_{33} \mathrm{~A}_{3}=0
\end{aligned}
$$

Where:

$$
\begin{aligned}
\mathrm{L}_{11} & =\mathrm{B}_{11} \mathrm{k}_{1}+\frac{2\left\{2 \mathrm{~B}_{33}+\mathrm{B}_{12}\right\}}{\beta^{2}} \mathrm{k}_{2}+\frac{\mathrm{B}_{22}}{\beta^{4}} \mathrm{k}_{3}+3 \frac{\mathrm{B}_{13}}{\beta} \mathrm{k}_{4}+3 \frac{\mathrm{B}_{23}}{\beta^{3}} \mathrm{k}_{5} \\
\mathrm{~L}_{12} & =\mathrm{B}_{11} \mathrm{~g}_{2} \mathrm{k}_{1}+\frac{\left\{2 \mathrm{~B}_{33}+\mathrm{B}_{12}\right\}}{\beta^{2}} \mathrm{~g}_{2} \mathrm{k}_{2}+2.25 \frac{\mathrm{B}_{13}}{\beta} \mathrm{g}_{2} \mathrm{k}_{4}+0.75 \frac{\mathrm{B}_{13}}{\beta} \mathrm{g}_{2} \mathrm{k}_{5} \\
\mathrm{~L}_{13} & =\frac{\left\{2 \mathrm{~B}_{33}+\mathrm{B}_{12}\right\}}{\beta^{2}} \mathrm{~g}_{2} \mathrm{k}_{2}+\frac{\mathrm{B}_{22}}{\beta^{4}} \mathrm{~g}_{2} \mathrm{k}_{3}+0.75 \frac{\mathrm{B}_{13}}{\beta} \mathrm{g}_{2} \mathrm{k}_{4}+2.25 \frac{\mathrm{B}_{23}}{\beta} \mathrm{g}_{2} \mathrm{k}_{5} \\
\mathrm{~L}_{12} & =\mathrm{B}_{11} \mathrm{~g}_{2} \mathrm{k}_{1}+\frac{\left\{2 \mathrm{~B}_{33}+\mathrm{B}_{12}\right\}}{\beta^{2}} \mathrm{~g}_{2} \mathrm{k}_{2}+2.25 \frac{\mathrm{B}_{13}}{\beta} \mathrm{g}_{2} \mathrm{k}_{4}+0.75 \frac{\mathrm{B}_{13}}{\beta} \mathrm{g}_{2} \mathrm{k}_{5} \\
\mathrm{~L}_{22} & =\mathrm{B}_{11} \mathrm{~g}_{3} \mathrm{k}_{1}+\frac{\mathrm{B}_{33}}{\beta^{2}} \mathrm{~g}_{3} \mathrm{k}_{2}+1.5 \frac{\mathrm{B}_{13}}{\beta} \mathrm{g}_{3} \mathrm{k}_{4}+\mathrm{B}_{44} \propto^{2} \mathrm{~g}_{4} \mathrm{k}_{6} \\
\mathrm{~L}_{23} & =\frac{\mathrm{B}_{12}}{\beta^{2}} \mathrm{~g}_{3} \mathrm{k}_{2}+\frac{\mathrm{B}_{33}}{\beta^{2}} \mathrm{~g}_{3} \mathrm{k}_{2}+0.75 \frac{\mathrm{B}_{13}}{\beta} \mathrm{g}_{3} \mathrm{k}_{4}+0.75 \frac{\mathrm{B}_{23}}{\beta^{3}} \mathrm{~g}_{3} \mathrm{k}_{5}+\propto^{2} \mathrm{~g}_{4} \frac{\mathrm{B}_{45}}{\beta} \mathrm{k}_{8} \\
\mathrm{~L}_{13} & =\frac{\left\{2 \mathrm{~B}_{33}+\mathrm{B}_{12}\right\}}{\mathrm{p}^{2}} \mathrm{~g}_{2} \mathrm{k}_{2}+\frac{\mathrm{B}_{22}}{\mathrm{p}^{4}} \mathrm{~g}_{2} \mathrm{k}_{3}+0.75 \frac{\mathrm{B}_{13}}{\mathrm{p}} \mathrm{g}_{2} \mathrm{k}_{4}+2.25 \frac{\mathrm{B}_{23}}{\mathrm{p}} \mathrm{g}_{2} \mathrm{k}_{5} \\
\mathrm{~L}_{23} & =\frac{\mathrm{B}_{12}}{\mathrm{p}^{2}} \mathrm{~g}_{3} \mathrm{k}_{2}+\frac{\mathrm{B}_{33}}{\mathrm{p}^{2}} \mathrm{~g}_{3} \mathrm{k}_{2}+0.75 \frac{\mathrm{B}_{13}}{\mathrm{p}} \mathrm{g}_{3} \mathrm{k}_{4}+0.75 \frac{\mathrm{B}_{23}}{\mathrm{p}^{3}} \mathrm{~g}_{3} \mathrm{k}_{5}+\propto^{2} \mathrm{~g}_{4} \frac{\mathrm{B}_{45}}{\mathrm{p}} \mathrm{k}_{8} \\
\mathrm{~L}_{33} & =\frac{\mathrm{B}_{33}}{\mathrm{p}^{2}} \mathrm{~g}_{3} \mathrm{k}_{2}+\frac{\mathrm{B}_{22}}{\mathrm{p}^{4}} \mathrm{~g}_{3} \mathrm{k}_{3}+1.5 \frac{\mathrm{B}_{23}}{\mathrm{p}^{3}} \mathrm{~g}_{3} \mathrm{k}_{5}+\propto^{2} \mathrm{~g}_{4} \frac{\mathrm{B}_{55}}{\mathrm{p}^{2}} \mathrm{k}_{7}
\end{aligned}
$$


Solving Equations 47 and 48 simultaneously gives:

$$
\begin{aligned}
& A_{2}=\left(\frac{L_{12} L_{33}-L_{13} L_{23}}{L_{22} L_{33}-L_{23} L_{23}}\right) A_{1}=P_{2} A_{1} \\
& A_{3}=\left(\frac{L_{13} L_{22}-L_{12} L_{23}}{L_{22} L_{33}-L_{23} L_{23}}\right) A_{1}=P_{3} A_{1}
\end{aligned}
$$

Substituting Equations 57 and 58 into Equation 46 gives:

$$
\mathrm{A}_{1}=\frac{\mathrm{q} a^{4}}{\mathrm{D}_{0}} \cdot \frac{\mathrm{k}_{8}}{\left(\mathrm{~L}_{11}-\mathrm{L}_{12} P_{2}-\mathrm{L}_{13} P_{3}\right)}=\frac{\mathrm{q} a^{4}}{\mathrm{D}_{0}} \cdot \mathrm{k}_{9}
$$

\section{Novel formulas for analysis}

Substituting Equation 60 into Equation 41a and substituting Equation 36 into the resulting equation and simplifying gives:

$$
w \frac{\mathrm{E}_{0} \mathrm{t}^{3}}{\mathrm{q} a^{4}}=12\left[1-\mu_{12} \mu_{21}\right] \cdot \mathrm{k}_{9} h
$$

Substituting Equations 41a, 42 and 43 into Equations 8c, 8d, 29, 30, 31, 32 and 33, where appropriate and simplifying gives:

$$
\begin{aligned}
& u \frac{\mathrm{E}_{0}}{\mathrm{q} a}\left(\frac{\mathrm{t}}{\mathrm{a}}\right)^{2}=12\left[1-\mu_{12} \mu_{21}\right] \cdot \mathrm{k}_{9} \cdot\left[-S+H P_{2}\right] \cdot \frac{\partial \mathrm{h}}{\partial \mathrm{R}} \\
& v \frac{\mathrm{E}_{0}}{q a}\left(\frac{\mathrm{t}}{\mathrm{a}}\right)^{2}=12\left[1-\mu_{12} \mu_{21}\right] \cdot \frac{\left[-S+H \cdot P_{3}\right]}{\beta} \cdot \frac{\partial h}{\partial Q} \cdot \mathrm{k}_{9} \\
& \frac{\sigma_{\mathrm{R}}}{\mathrm{q}}\left(\frac{t}{a}\right)^{2}=12 . \mathrm{k}_{9}\left(\mathrm{~B}_{11}\left[H P_{2}-S\right] \frac{\partial^{2} h}{\partial R^{2}}+\frac{\mathrm{B}_{12}}{\beta^{2}}\left[H P_{3}-S\right] \frac{\partial^{2} h}{\partial Q^{2}}\right. \\
& \left.+\frac{B_{13}}{\beta} H\left(P_{2}+P_{3}-2 S\right) \frac{\partial^{2} h}{\partial R \partial Q}\right) \\
& \frac{\sigma_{\mathrm{Q}}}{\mathrm{q}}\left(\frac{t}{a}\right)^{2}=12 . \mathrm{k}_{\mathrm{9}} \cdot\left(\mathrm{B}_{21}\left[H P_{2}-S\right] \frac{\partial^{2} h}{\partial R^{2}}+\frac{\mathrm{B}_{22}}{\beta^{2}}\left[H P_{3}-S\right] \frac{\partial^{2} h}{\partial Q^{2}}+\frac{B_{23}}{\beta} H\left(P_{2}+P_{3}\right)\right. \\
& \left.-2 S \frac{\partial^{2} h}{\partial R \partial Q}\right) \\
& \frac{\tau_{\mathrm{RQ}}}{\mathrm{q}}\left(\frac{t}{a}\right)^{2}=12 \mathrm{k}_{9} \cdot\left(\mathrm{B}_{31} \cdot\left[H P_{2}-S\right] \frac{\partial^{2} h}{\partial R^{2}}+\frac{\mathrm{B}_{32}}{\beta^{2}} \cdot\left[H P_{3}-S\right] \frac{\partial^{2} h}{\partial Q^{2}}\right. \\
& \left.+\frac{\mathrm{B}_{33}}{\beta} \cdot H\left(P_{2}+P_{3}-2 S\right) \frac{\partial^{2} h}{\partial R \partial Q}\right) \\
& \overline{\tau_{\mathrm{RS}}}=\frac{\tau_{\mathrm{RS}}}{\mathrm{q}}\left(\frac{t}{a}\right)^{3}=12 \mathrm{k}_{9}\left(\mathrm{~B}_{44} \cdot P_{2} \cdot \frac{\partial H}{\partial S}\right) \cdot \frac{\partial \mathrm{h}}{\partial \mathrm{R}} \\
& \overline{\tau_{\mathrm{QS}}}=\frac{\tau_{\mathrm{QS}}}{\mathrm{q}}\left(\frac{t}{a}\right)^{3}=12 \cdot \mathrm{k}_{9} \cdot\left(\mathrm{B}_{55} \cdot \frac{P_{3}}{\beta} \cdot \frac{\partial H}{\partial S}\right) \cdot \frac{\partial \mathrm{h}}{\partial \mathrm{Q}}
\end{aligned}
$$

\section{Typical example problems for SSSS thick anisotropic plate}

The formulas were used to analyze typical anisotropic rectangular thick plates. The numerical values for In-plane displacements, $\mathrm{u}$ and $\mathrm{v}$, out-plane displacement (central deflection), w, in-plane stresses, $\sigma_{\mathrm{x}}, \sigma_{\mathrm{y}}$ and $\tau_{\mathrm{xy}}$, and out-plane stresses, $\tau_{\mathrm{xz}}$ and $\tau_{\mathrm{yz}}$, were determined for angles fiber orientations of $0^{0}, 15^{\circ}, 30^{\circ}$, $45^{\circ}, 60^{\circ}, 75^{\circ}$ and $90^{\circ}$ at span to thickness ration $(\alpha), 5$, $10,20,30,40,50,60,70,80,90$ and 100 for plate that is simply supported at all edges (SSSS). The plate was analyzed at various meaningful points along the length, width and depth axis; in-plane displacement ( $\mathrm{u}$ and $\mathrm{v}$ ) at $(\mathrm{x}=0.5, \mathrm{y}=0.5, \mathrm{z}=0.5)$, transverse displacement $(w)$ at $(x=0.5, y=0.5, z=0.5)$, In- plane normal stresses $\left(\sigma_{\mathrm{x}}\right.$ and $\left.\sigma_{\mathrm{y}}\right)$ at $(\mathrm{x}=0.5, \mathrm{y}=$ $0.5, \mathrm{z}=0.5$ or $\mathrm{z}=0.25)$, in-plane shear stress $\left(\tau_{\mathrm{xy}}\right)$ at $(\mathrm{x}=0, \mathrm{y}=0, \mathrm{z}=0.5)$, out-plane shear stress $\left(\tau_{\mathrm{xz}}\right)$ at $(\mathrm{x}=0, \mathrm{y}=0.5, \mathrm{z}=0.5)$ and outplane shear stress $\left(\tau_{\mathrm{yz}}\right)$ at $(\mathrm{x}=0.5, \mathrm{y}=0, \mathrm{z}=0.5)$ respectively. The plate is subjected to uniformly distributed load. The following non dimensionalizations that were applied by $[7,8,9,10,11,12,13]$ were used to present the results; $\left[\overline{\mathrm{w}}=\mathrm{w} \frac{\mathrm{E}_{0} \mathrm{t}^{3}}{\mathrm{q} a^{4}} \mathrm{x} 100, \overline{\mathrm{u}}, \overline{\mathrm{v}}=\right.$ $\mathrm{u}, \mathrm{v} \frac{\mathrm{E}_{0} \mathrm{t}^{2}}{\mathrm{q} a^{3}},\left(\overline{\sigma_{\mathrm{xx}}}, \overline{\sigma_{\mathrm{yy}}}, \overline{\tau_{\mathrm{xy}}}\right)=\left(\frac{\sigma_{\mathrm{x}}, \sigma_{\mathrm{y}}, \tau_{\mathrm{xy}} \mathrm{t}^{2}}{\mathrm{q} a^{2}}\right),\left(\overline{\tau_{\mathrm{xz}}}, \overline{\tau_{\mathrm{yz}}}\right)=$ $\left.\left(\frac{\tau_{\mathrm{xz}} \mathrm{t}}{\mathrm{q} a}\right)\right]$. The material properties used were as follows; for Table 1 to $7, \quad\left(E_{1} / E_{2}=25, G_{12} / E_{2}=\right.$ 
$\left.0.5, \mathrm{G}_{13} / \mathrm{E}_{2}=0.5, G_{23} / \mathrm{E}_{2}=0.2, \mathrm{v}_{12}=0.25\right)$ and for Table $8, \quad\left(\mathrm{E}_{1}=\mathrm{E}_{2}=210 \mathrm{GPa}, \mathrm{v}_{12}=0.3, \mathrm{G}=\right.$ $\left.\frac{E}{2(1+\mu)}\right)$ respectively.

i. Analyze an orthotropic thick square SSSS plate with the following information:

$(\mathrm{E} 1=25 ; \mathrm{E} 2=1 ; \mathrm{G} 12=0.5 ; \mathrm{G} 13=$

$0.5 ; \mathrm{G} 23=0.2, \mu 12=0.25$ )

ii. Analyze an orthotropic thick square SSSS plate with the following information:

$\left(\mathrm{E}_{1}=\mathrm{E}_{2}=210 \mathrm{GPa}, \mathrm{v}_{12}=0.3, \mathrm{G}=\frac{E}{2(1+\mu)}\right)$

$$
\begin{aligned}
& u \frac{\mathrm{E}_{0}}{\mathrm{q} a}\left(\frac{\mathrm{t}}{\mathrm{a}}\right)^{2}=12\left[1-\mu_{12} \mu_{21}\right] \cdot \mathrm{k}_{9} \cdot\left[-S+H P_{2}\right] \cdot \frac{\partial \mathrm{h}}{\partial \mathrm{R}} \\
& v \frac{\mathrm{E}_{0}}{q a}\left(\frac{\mathrm{t}}{\mathrm{a}}\right)^{2}=12\left[1-\mu_{12} \mu_{21}\right] \cdot \frac{\left[-S+H \cdot P_{3}\right]}{\beta} \cdot \frac{\partial h}{\partial Q} \cdot \mathrm{k}_{9} \\
& \frac{\sigma_{\mathrm{R}}}{\mathrm{q}}\left(\frac{t}{a}\right)^{2}=12 . \mathrm{k}_{9}\left(\mathrm{~B}_{11}\left[H P_{2}-S\right] \frac{\partial^{2} h}{\partial R^{2}}+\frac{\mathrm{B}_{12}}{\beta^{2}}\left[H P_{3}-S\right] \frac{\partial^{2} h}{\partial Q^{2}}\right. \\
& \left.+\frac{B_{13}}{\beta} H\left(P_{2}+P_{3}-2 S\right) \frac{\partial^{2} h}{\partial R \partial Q}\right) \\
& \frac{\sigma_{\mathrm{Q}}}{\mathrm{q}}\left(\frac{t}{a}\right)^{2}=12 \cdot \mathrm{k}_{9} \cdot\left(\mathrm{B}_{21}\left[H P_{2}-S\right] \frac{\partial^{2} h}{\partial R^{2}}+\frac{\mathrm{B}_{22}}{\beta^{2}}\left[H P_{3}-S\right] \frac{\partial^{2} h}{\partial Q^{2}}+\frac{B_{23}}{\beta} H\left(P_{2}+P_{3}\right)\right. \\
& \left.-2 S \frac{\partial^{2} h}{\partial R \partial Q}\right) \\
& \frac{\tau_{\mathrm{RQ}}}{\mathrm{q}}\left(\frac{t}{a}\right)^{2}=12 \mathrm{k}_{9} \cdot\left(\mathrm{B}_{31} \cdot\left[H P_{2}-S\right] \frac{\partial^{2} h}{\partial R^{2}}+\frac{\mathrm{B}_{32}}{\beta^{2}} \cdot\left[H P_{3}-S\right] \frac{\partial^{2} h}{\partial Q^{2}}\right. \\
& \left.+\frac{\mathrm{B}_{33}}{\beta} \cdot H\left(P_{2}+P_{3}-2 S\right) \frac{\partial^{2} h}{\partial R \partial Q}\right) \\
& \overline{\tau_{\mathrm{RS}}}=\frac{\tau_{\mathrm{RS}}}{\mathrm{q}}\left(\frac{t}{a}\right)^{3}=12 \mathrm{k}_{9}\left(\mathrm{~B}_{44} \cdot P_{2} \cdot \frac{\partial H}{\partial S}\right) \cdot \frac{\partial \mathrm{h}}{\partial \mathrm{R}} \\
& \overline{\tau_{\mathrm{QS}}}=\frac{\tau_{\mathrm{QS}}}{\mathrm{q}}\left(\frac{t}{a}\right)^{3}=12 \cdot \mathrm{k}_{9} \cdot\left(\mathrm{B}_{55} \cdot \frac{P_{3}}{\beta} \cdot \frac{\partial H}{\partial S}\right) \cdot \frac{\partial \mathrm{h}}{\partial \mathrm{Q}}
\end{aligned}
$$

\section{RESULTS AND DISCUSSIONS}

\section{Presentation of Results}

The results of SSSS rectangular thick anisotropic plate were presented here.

\section{Displacements and stresses formulas}

The novel formulas derived in the present study for the determination of displacements and stresses are presented as follows:
69

\section{RESULTS OF NUMERICAL PROBLEMS} (1) to (7).

The numerical values for displacements ( $\mathrm{u}, \mathrm{v}$ and $\mathrm{w})$ and stresses $\left(\sigma_{\mathrm{x}}, \sigma_{\mathrm{y}}, \tau_{\mathrm{xy}}, \tau_{\mathrm{xz}}\right.$ and $\left.\tau_{\mathrm{yz}}\right)$ are presented on Tables

Table-1: Displacements and stresses for SSSS anisotropic rectangular thick plate for $0^{0} @(\alpha=5$ to $100, \beta=$

\begin{tabular}{|c|c|c|c|c|c|c|c|c|}
\hline$\alpha$ & $\overline{\mathbf{w}}$ & $\overline{\mathbf{u}}$ & $\overline{\mathbf{v}}$ & $\overline{\boldsymbol{\sigma}_{\mathbf{x x}}}$ & $\overline{\boldsymbol{\sigma}_{\mathbf{y y}}}$ & $\overline{\boldsymbol{\tau}_{\mathbf{x y}}}$ & $\overline{\boldsymbol{\tau}_{\mathbf{x z}}}$ & $\overline{\boldsymbol{\tau}_{\mathbf{y z}}}$ \\
\hline 5 & 0.0180 & -0.32005 & -0.60615 & 0.980772 & 0.0364545 & 0.0592765 & 0.59966 & 0.06820 \\
\hline 10 & 0.01005 & -1.15660 & -1.51589 & 0.881023 & 0.0259871 & 0.0427599 & 0.67721 & 0.05531 \\
\hline 20 & 0.00775 & -4.4924 & -4.87597 & 0.853597 & 0.0222705 & 0.0374734 & 0.70067 & 0.05011 \\
\hline 30 & 0.00731 & -10.0509 & -10.4394 & 0.848404 & 0.0215162 & 0.0364274 & 0.70524 & 0.04903 \\
\hline 40 & 0.00715 & -17.8329 & -18.2231 & 0.846577 & 0.0212470 & 0.0360560 & 0.70686 & 0.04864 \\
\hline 50 & 0.00708 & -27.8382 & -28.2292 & 0.845730 & 0.0211214 & 0.0358832 & 0.70761 & 0.04846 \\
\hline 60 & 0.00704 & -40.0669 & -40.4583 & 0.845270 & 0.0210529 & 0.0357890 & 0.70802 & 0.04836 \\
\hline 70 & 0.00701 & -54.5190 & -54.9107 & 0.844992 & 0.0210115 & 0.0357321 & 0.70827 & 0.04830 \\
\hline 80 & 0.0070 & -71.1945 & -71.5863 & 0.844811 & 0.0209846 & 0.0356952 & 0.70843 & 0.04826 \\
\hline 90 & 0.00699 & -90.0934 & -90.4853 & 0.844688 & 0.0209662 & 0.0356699 & 0.70854 & 0.04823 \\
\hline 100 & 0.00698 & -111.216 & -111.608 & 0.844599 & 0.0209530 & 0.0356517 & 0.70862 & 0.04821 \\
\hline
\end{tabular}


Table-2: Displacements and stresses for SSSS anisotropic rectangular thick plate for

\begin{tabular}{|l|c|c|c|c|c|c|c|c|}
\multicolumn{10}{|c}{$\mathbf{1 5}^{\mathbf{0}} @(\boldsymbol{\alpha}=\mathbf{5}$ to $\mathbf{1 0 0} \boldsymbol{\beta}=\mathbf{1})$} \\
\hline $\boldsymbol{\alpha}$ & $\overline{\mathbf{w}}$ & $\overline{\mathbf{u}}$ & $\overline{\mathbf{v}}$ & $\overline{\boldsymbol{\sigma}_{\mathbf{x x}}}$ & $\overline{\boldsymbol{\sigma}_{\mathbf{y y}}}$ & $\overline{\boldsymbol{\tau}_{\mathbf{x y}}}$ & $\overline{\boldsymbol{\tau}_{\mathbf{x z}}}$ & $\overline{\boldsymbol{\tau}_{\mathbf{y z}}}$ \\
\hline 5 & 0.01245 & -0.2212 & -0.379625 & 0.661806 & 0.0977385 & 0.1517064 & 0.41532 & 0.071053 \\
\hline 10 & 0.00791 & -0.9118 & -1.103948 & 0.658215 & 0.0849413 & 0.1272449 & 0.53056 & 0.096888 \\
\hline 20 & 0.00634 & -3.6773 & -3.879932 & 0.656304 & 0.0807469 & 0.1192681 & 0.57014 & 0.106484 \\
\hline 30 & 0.00602 & -8.2868 & -8.491418 & 0.655874 & 0.0799134 & 0.1176856 & 0.57813 & 0.108468 \\
\hline 40 & 0.00591 & -14.740 & -14.94539 & 0.655717 & 0.0796172 & 0.1171235 & 0.58098 & 0.109179 \\
\hline 50 & 0.00586 & -23.037 & -23.24277 & 0.655643 & 0.0794793 & 0.1168619 & 0.58231 & 0.109512 \\
\hline 60 & 0.00583 & -33.178 & -33.38378 & 0.655603 & 0.0794042 & 0.1167193 & 0.58304 & 0.109693 \\
\hline 70 & 0.00581 & -45.163 & -45.36852 & 0.655578 & 0.0793588 & 0.1166332 & 0.58347 & 0.109803 \\
\hline 80 & 0.00580 & -58.991 & -59.19701 & 0.655562 & 0.0793293 & 0.1165773 & 0.58376 & 0.109874 \\
\hline 90 & 0.00579 & -74.663 & -74.86926 & 0.655551 & 0.0793091 & 0.1165390 & 0.58395 & 0.109923 \\
\hline 100 & 0.00579 & -92.179 & -92.38529 & 0.655543 & 0.0792946 & 0.1165115 & 0.58409 & 0.109958 \\
\hline
\end{tabular}

Table-3: Displacements and stresses for SSSS anisotropic rectangular thick plate for $30^{0} @(\alpha=5$ to $100, \beta=1)$

\begin{tabular}{|l|c|c|c|c|c|c|c|c|}
\hline \multicolumn{1}{|c|}{} & $\overline{\mathbf{w}}$ & $\overline{\mathbf{u}}$ & $\overline{\mathbf{v}}$ & $\overline{\boldsymbol{\sigma}_{\mathbf{x x}}}$ & $\overline{\boldsymbol{\sigma}_{\mathbf{y y}}}$ & $\overline{\boldsymbol{\tau}_{\mathbf{x y}}}$ & $\overline{\boldsymbol{\tau}_{\mathbf{x z}}}$ & $\overline{\boldsymbol{\tau}_{\mathbf{y z}}}$ \\
\hline 5 & 0.00774 & -0.15608 & -0.179078 & 0.374328 & 0.1433016 & 0.2109912 & 0.23050 & 0.07840 \\
\hline 10 & 0.00568 & -0.68334 & -0.656346 & 0.391837 & 0.1468940 & 0.2108444 & 0.33873 & 0.15169 \\
\hline 20 & 0.00471 & -2.75267 & -2.695600 & 0.396415 & 0.1489391 & 0.2143670 & 0.38959 & 0.19008 \\
\hline 30 & 0.00448 & -6.19068 & -6.126367 & 0.397230 & 0.1494259 & 0.2153889 & 0.40116 & 0.19905 \\
\hline 40 & 0.00440 & -11.0020 & -10.93497 & 0.397511 & 0.1496063 & 0.2157821 & 0.40540 & 0.20236 \\
\hline 50 & 0.00436 & -17.1873 & -17.11908 & 0.397641 & 0.1496919 & 0.2159707 & 0.40740 & 0.20393 \\
\hline 60 & 0.00434 & -24.7471 & -24.67808 & 0.397711 & 0.1497388 & 0.2160750 & 0.40850 & 0.20479 \\
\hline 70 & 0.00433 & -33.6811 & -33.61173 & 0.397752 & 0.1497673 & 0.2161385 & 0.40916 & 0.20531 \\
\hline 80 & 0.00432 & -43.9896 & -43.91996 & 0.397780 & 0.1497858 & 0.2161799 & 0.40960 & 0.20565 \\
\hline 90 & 0.00432 & -55.6726 & -55.60270 & 0.397799 & 0.1497986 & 0.2162085 & 0.40989 & 0.20588 \\
\hline 100 & 0.00431 & -68.7299 & -68.65994 & 0.397812 & 0.1498077 & 0.2162290 & 0.41011 & 0.20605 \\
\hline
\end{tabular}

Table-4: Displacements and stresses for SSSS anisotropic rectangular thick plate for values; $\mathbf{4 5}^{0} @$ $(\alpha=5$ to $100, \beta=1)$

\begin{tabular}{|l|c|c|c|c|c|c|c|c|}
\hline $\boldsymbol{\alpha}$ & $\overline{\mathbf{w}}$ & $\overline{\mathbf{u}}$ & $\overline{\mathbf{v}}$ & $\overline{\boldsymbol{\sigma}_{\mathbf{x x}}}$ & $\overline{\boldsymbol{\sigma}_{\mathbf{y y}}}$ & $\overline{\boldsymbol{\tau}_{\mathbf{x y}}}$ & $\overline{\boldsymbol{\tau}_{\mathbf{x z}}}$ & $\overline{\boldsymbol{\tau}_{\mathbf{y z}}}$ \\
\hline 5 & 0.00680 & -0.19077 & -0.089217 & 0.229241 & 0.2170548 & 0.22904 & 0.12201 & 0.10974 \\
\hline 10 & 0.00521 & -0.68901 & -0.484018 & 0.236799 & 0.2306494 & 0.23989 & 0.21532 & 0.20913 \\
\hline 20 & 0.00425 & -2.54258 & -2.275540 & 0.241002 & 0.2389991 & 0.24634 & 0.27041 & 0.26839 \\
\hline 30 & 0.00402 & -5.59693 & -5.314405 & 0.242033 & 0.2410916 & 0.24794 & 0.28410 & 0.28315 \\
\hline 40 & 0.00393 & -9.86670 & -9.578364 & 0.242420 & 0.2418793 & 0.24855 & 0.28925 & 0.28870 \\
\hline 50 & 0.00389 & -15.3545 & -15.06343 & 0.242604 & 0.2422543 & 0.24883 & 0.29169 & 0.29135 \\
\hline 60 & 0.00386 & -22.0612 & -21.76853 & 0.242705 & 0.2424610 & 0.24899 & 0.29305 & 0.29280 \\
\hline 70 & 0.00385 & -29.9868 & -29.69326 & 0.242766 & 0.2425866 & 0.24909 & 0.29387 & 0.29368 \\
\hline 80 & 0.00384 & -39.1316 & -38.83746 & 0.242806 & 0.2426685 & 0.24915 & 0.29440 & 0.29426 \\
\hline 90 & 0.00383 & -49.4956 & -49.20106 & 0.242834 & 0.2427248 & 0.24919 & 0.29477 & 0.29466 \\
\hline 100 & 0.00383 & -61.0789 & -60.78399 & 0.242854 & 0.2427652 & 0.24922 & 0.29503 & 0.29494 \\
\hline
\end{tabular}

Table-5: Displacements and stresses for SSSS anisotropic rectangular thick plate for $60^{0} @(\alpha=5$ to $100, \beta=1)$

\begin{tabular}{|l|c|c|c|c|l|l|c|c|}
\hline $\boldsymbol{\alpha}$ & $\overline{\mathbf{w}}$ & $\overline{\mathbf{u}}$ & $\overline{\mathbf{v}}$ & $\overline{\boldsymbol{\sigma}_{\mathbf{x x}}}$ & $\overline{\boldsymbol{\sigma}_{\mathbf{y y}}}$ & $\overline{\boldsymbol{\tau}_{\mathbf{x y}}}$ & $\overline{\boldsymbol{\tau}_{\mathbf{x z}}}$ & $\overline{\boldsymbol{\tau}_{\mathbf{y z}}}$ \\
\hline 5 & 0.00896 & -0.30878 & -0.086577 & 0.144820 & 0.3249868 & 0.2488920 & 0.07430 & 0.16304 \\
\hline 10 & 0.00643 & -0.93244 & -0.533919 & 0.147495 & 0.364930 & 0.2307812 & 0.14551 & 0.29731 \\
\hline 20 & 0.00497 & -3.05655 & -2.556298 & 0.149099 & 0.3875076 & 0.2208424 & 0.18736 & 0.37509 \\
\hline 30 & 0.00461 & -6.50804 & -5.982777 & 0.149497 & 0.3930315 & 0.2184275 & 0.19769 & 0.39423 \\
\hline 40 & 0.00448 & -11.3243 & -10.78976 & 0.149646 & 0.3950991 & 0.2175250 & 0.20156 & 0.40140 \\
\hline 50 & 0.00441 & -17.5121 & -16.97309 & 0.149717 & 0.3960814 & 0.2170964 & 0.20341 & 0.40481 \\
\hline 60 & 0.00438 & -25.0731 & -24.53166 & 0.149757 & 0.3966220 & 0.2168606 & 0.20442 & 0.40668 \\
\hline 70 & 0.00436 & -34.0080 & -33.46506 & 0.149780 & 0.3969503 & 0.2167174 & 0.20504 & 0.40782 \\
\hline 80 & 0.00434 & -44.3171 & -43.77311 & 0.149796 & 0.3971644 & 0.2166241 & 0.20544 & 0.40857 \\
\hline 90 & 0.00433 & -56.0004 & -55.45574 & 0.149806 & 0.3973116 & 0.2165599 & 0.20571 & 0.40908 \\
\hline 100 & 0.00432 & -69.0580 & -68.51289 & 0.149814 & 0.3974171 & 0.2165139 & 0.20591 & 0.40945 \\
\hline
\end{tabular}


Table-6: Displacements and stresses for SSSS anisotropic rectangular thick plate for

\begin{tabular}{|c|c|c|c|c|c|c|c|c|}
\hline \multicolumn{10}{|c|}{$\mathbf{7 5}^{\mathbf{0}} @(\boldsymbol{\alpha}=\mathbf{5}$ to $\mathbf{1 0 0}, \boldsymbol{\beta}=\mathbf{1})$} \\
\hline $\mathbf{\alpha}$ & $\overline{\mathbf{w}}$ & $\overline{\mathbf{u}}$ & $\overline{\mathbf{v}}$ & $\overline{\boldsymbol{\sigma}_{\mathbf{x x}}}$ & $\overline{\boldsymbol{\sigma}_{\mathbf{y y}}}$ & $\overline{\boldsymbol{\tau}_{\mathbf{x y}}}$ & $\overline{\boldsymbol{\tau}_{\mathbf{x z}}}$ & $\overline{\boldsymbol{\tau}_{\mathbf{y z}}}$ \\
\hline 10 & 0.01683 & -0.61624 & -0.188913 & 0.123480 & 0.6256783 & 0.2033115 & 0.08573 & 0.29069 \\
\hline 20 & 0.01018 & -1.56106 & -0.851163 & 0.097464 & 0.6419197 & 0.1522786 & 0.10206 & 0.46676 \\
\hline 30 & 0.00706 & -4.44752 & -3.602851 & 0.084644 & 0.6513665 & 0.1270507 & 0.10788 & 0.54996 \\
\hline 40 & 0.00610 & -9.08430 & -8.209154 & 0.081723 & 0.6535931 & 0.1212998 & 0.10910 & 0.56872 \\
\hline 50 & 0.00598 & -23.8493 & -22.95775 & 0.080146 & 0.6548053 & 0.1181932 & 0.10974 & 0.57884 \\
\hline 60 & 0.00591 & -33.9927 & -33.09829 & 0.079869 & 0.6550188 & 0.1176475 & 0.10985 & 0.58061 \\
\hline 70 & 0.00587 & -45.9789 & -45.08273 & 0.079701 & 0.6551483 & 0.1173168 & 0.10992 & 0.58169 \\
\hline 80 & 0.00585 & -59.8083 & -58.91103 & 0.079592 & 0.6552327 & 0.1171015 & 0.10996 & 0.58239 \\
\hline 90 & 0.00583 & -75.4812 & -74.58315 & 0.079517 & 0.6552906 & 0.1169536 & 0.10999 & 0.58287 \\
\hline 100 & 0.00582 & -92.9977 & -92.09909 & 0.079463 & 0.6553322 & 0.1168476 & 0.11001 & 0.58321 \\
\hline
\end{tabular}

Table-7: Displacements and stresses for SSSS anisotropic rectangular thick plate for values; $90^{0} @(\alpha=$ 5 to $100, \beta=1)$

\begin{tabular}{|l|c|c|c|c|c|c|c|c|}
\hline \multicolumn{1}{|c|}{$\boldsymbol{\alpha}$} & $\overline{\mathbf{w}}$ & $\overline{\mathbf{u}}$ & $\overline{\mathbf{v}}$ & $\overline{\boldsymbol{\sigma}_{\mathbf{x x}}}$ & $\overline{\boldsymbol{\sigma}_{\mathbf{y y}}}$ & $\overline{\boldsymbol{\tau}_{\mathbf{x y}}}$ & $\overline{\boldsymbol{\tau}_{\mathbf{x z}}}$ & $\overline{\boldsymbol{\tau}_{\mathbf{y z}}}$ \\
\hline 5 & 0.02864 & -1.06997 & -0.350592 & 0.139262 & 1.08659 & 0.0909160 & 0.11324 & 0.47692 \\
\hline 10 & 0.01410 & -2.20768 & -1.204006 & 0.075449 & 0.92186 & 0.0545870 & 0.07184 & 0.63094 \\
\hline 20 & 0.00889 & -5.65573 & -4.546235 & 0.051070 & 0.86518 & 0.0408079 & 0.05475 & 0.68760 \\
\hline 30 & 0.00783 & -11.2375 & -10.10619 & 0.045995 & 0.85368 & 0.0379445 & 0.05113 & 0.69929 \\
\hline 40 & 0.00745 & -19.0278 & -17.88861 & 0.044173 & 0.84957 & 0.0369165 & 0.04983 & 0.70348 \\
\hline 50 & 0.00727 & -29.0370 & -27.89414 & 0.043321 & 0.84765 & 0.0364360 & 0.04922 & 0.70544 \\
\hline 60 & 0.00717 & -41.2678 & -40.12295 & 0.042856 & 0.84660 & 0.0361737 & 0.04889 & 0.70651 \\
\hline 70 & 0.00711 & -55.7212 & -54.57511 & 0.042575 & 0.84597 & 0.0360151 & 0.04869 & 0.70715 \\
\hline 80 & 0.00707 & -72.3975 & -71.25064 & 0.042392 & 0.84556 & 0.0359121 & 0.04856 & 0.70757 \\
\hline 90 & 0.00705 & -91.2970 & -90.14955 & 0.042267 & 0.84528 & 0.0358413 & 0.04847 & 0.70786 \\
\hline 100 & 0.00703 & -112.419 & -111.2718 & 0.042177 & 0.84508 & 0.0357906 & 0.04840 & 0.70807 \\
\hline
\end{tabular}

Table-8: Comparison of present study results with various results from isotropic square SSSS plate subjected to uniformly distributed load

\begin{tabular}{|l|l|l|l|l|l|l|l|}
\hline $\mathbf{a} / \mathbf{t}$ & Author & Theory & \multicolumn{1}{|c}{$\overline{\mathbf{u}}$} & $\overline{\mathbf{w}}$ & $\overline{\boldsymbol{\sigma}_{\mathbf{x x}}}$ & $\overline{\mathbf{\tau}_{\mathbf{x y}}}$ & $\overline{\mathbf{\tau}_{\mathbf{x z}}}$ \\
\hline 4 & Present & TOSDT & 0.07213 & 5.4893 & 0.3091 & 0.1775 & 0.2898 \\
\hline & Sayyad et al. & ESDT & 0.079 & 5.816 & 0.300 & 0.223 & 0.481 \\
\hline & \%difference & & $\mathbf{- 9 . 5 2 \%}$ & $\mathbf{- 5 . 9 5 \%}$ & $\mathbf{2 . 9 4 \%}$ & $\mathbf{- 2 5 . 6 \%}$ & $\mathbf{- 6 5 . 9 \%}$ \\
\hline & Reddy & HSDT & 0.079 & 5.869 & 0.299 & 0.218 & 0.482 \\
\hline & Ghugal\&Sayyad & TSDT & 0.074 & 5.680 & 0.318 & 0.208 & 0.483 \\
\hline & Ghugal\&Pawar & HPSDT & 0.079 & 5.858 & 0.297 & 0.185 & 0.477 \\
\hline & Mindlin & FSDT & 0.074 & 5.633 & 0.287 & 0.195 & 0.330 \\
\hline & \%difference & & $\mathbf{- 2 . 5 9 \%}$ & $\mathbf{- 2 . 6 1 \%}$ & $\mathbf{7 . 1 4 \%}$ & $\mathbf{- 9 . 8 5 \%}$ & $\mathbf{- 1 3 . 8 \%}$ \\
\hline & Kirchhoff & CPT & 0.074 & 4.436 & 0.287 & 0.195 & - \\
\hline & Pagano & Elasticity & 0.072 & 5.694 & 0.307 & - & 0.460 \\
\hline 10 & Present & TSDT & 0.07225 & 4.6881 & 0.3097 & 0.1775 & 0.3177 \\
\hline & Sayyad et al. & ESDT & 0.075 & 4.658 & 0.289 & 0.204 & 0.494 \\
\hline & \%error & & $\mathbf{- 3 . 7 3 \%}$ & $\mathbf{0 . 6 4 2 \%}$ & $\mathbf{6 . 6 8 \%}$ & $\mathbf{- 1 4 . 6 \%}$ & $\mathbf{- 5 5 . 3 \%}$ \\
\hline & Reddy & HSDT & 0.075 & 4.666 & 0.289 & 0.203 & 0.492 \\
\hline & Ghugal\&Sayyad & TSDT & 0.073 & 4.625 & 0.307 & 0.195 & 0.504 \\
\hline & Ghugal\&Pawar & HPSDT & 0.074 & 4.665 & 0.289 & 0.193 & 0.489 \\
\hline & Mindlin & FSDT & 0.074 & 4.670 & 0.287 & 0.195 & 0.330 \\
\hline & \%error & & $\mathbf{- 2 . 3 5 \%}$ & $\mathbf{0 . 3 8 \%}$ & $\mathbf{7 . 3 3 \%}$ & $\mathbf{- 9 . 5 5 \%}$ & $\mathbf{- 3 . 7 7 \%}$ \\
\hline & Kirchhoff & CPT & 0.074 & 4.436 & 0.287 & 0.195 & - \\
\hline & Pagano & Elasticity & 0.073 & 4.639 & 0.289 & - & 0.487 \\
\hline
\end{tabular}




\section{RESULTS ANALYSIS}

\section{Displacements and stresses formulas}

The combination of the elastic equations, displacement functions equations, stiffness equations, governing equations and the compatibility equations yielded the required formulas for the calculation of displacements and stresses. The displacements follows similar pattern and they are generally related by the following terms $\left[12\left(k_{9}\right)\left(\frac{\mathrm{a}}{\mathrm{t}}\right)^{2} \cdot \frac{\mathrm{q}}{E_{0}} \cdot\left(1-\mu_{12} \cdot \mu_{21}\right)\right]$ Where $\frac{\mathrm{a}}{\mathrm{t}}$ is the ratio of span to thickness which is majorly used to classify the plate, $q$ is the pure bending loading on the plate, $E_{0}$ is the elastic modulus while $k_{9}$ is the stiffness coefficient which is calculated by dividing $\mathrm{k}_{8}$ with a combination of different parameters as shown in equations 49 to 60 . Although the in-plane displacements, $\mathrm{u}$ and $\mathrm{v}$, are more closely related with the different being the derivative $\left(\frac{\mathrm{dh}}{\mathrm{dR}}, \frac{\mathrm{dh}}{\mathrm{dQ}}\right)$ and aspect ratio $\left(P_{2}, P_{3}\right)$. When these displacement formulas are applied in a problem, the values obtained with the formulas of $\mathrm{u}$ and $\mathrm{v}$ are more closely related than the values obtained with the out-plane displacement $\mathrm{w}$.

The in-plane stresses $\left(\sigma_{R R}, \sigma_{Q Q}, \tau_{R Q}\right)$ have these terms in common, $\left[12 \mathrm{q} .\left(\frac{a}{t}\right)^{2}\left(k_{9}\right),\left(\mathrm{HP}_{2}-\right.\right.$ $S) \cdot \frac{\partial^{2} \mathrm{~h}}{\partial R^{2}},\left(\mathrm{HP}_{3}-S\right) \cdot \frac{\partial^{2} \mathrm{~h}}{\partial Q^{2}}$ and $\left.H\left(\mathrm{P}_{2}+\mathrm{P}_{3}-2 S\right) \cdot \frac{\partial^{2} \mathrm{~h}}{\partial \mathrm{R} \partial Q}\right]$. The common terms improves the applicability and usability of the solutions for easy solution of thick anisotropic plate. Also the out-plane displacement $\left(\tau_{R s}\right.$ $\left.\& \tau_{Q S}\right)$ have $\left[12 \mathrm{q} .\left(\mathrm{k}_{9}\right)\left(\frac{\mathrm{a}}{\mathrm{t}}\right)^{3} \cdot \frac{\partial \mathrm{H}}{\partial \mathrm{S}}\right]$ in common. These formulas are new and very easy to apply when analyzing thick anisotropic rectangular plate. It only requires the user to substitute the formulas data as provided in the problem at hand. These equations are the novel equations for displacements and stresses used for thick anisotropic rectangular plate analysis.

\section{Numerical values of SSSS plate at angle fiber orientation of $0^{0}$}

From Table 4.2, it is observed that out-plane displacement values (w) decreases toward the positive direction as the thickness of the plate decreases while the in-plane displacements ( $\mathrm{u} \& \mathrm{v}$ ) decrease toward the negative direction as the thickness of the plate decreases. The decreases were very visible at the thick plate zone ( $\alpha=5$ to 10$)$ but becomes very small and slightly negligible at the thin plate zone ( $\alpha=20$ to 100$)$. This is a confirmation that the effects of the displacement are more on thick plate than thin plate. The in-plane stresses, $\overline{\sigma_{x x}}, \overline{\sigma_{y y}}$, and $\tau_{x y}$ decrease as the plate thickness decreases. A close look will reveal a sharp decrease at thick and moderately thick plate section while the thin plate section decreased lightly. The out-plane stress $\tau_{x z}$ increases as the plate thickness decreases while the out-plane stress $\tau_{y z}$ decreases as the plate thickness decreases. This decrease of displacements values and increase and decrease of the stresses values of the plate can be explained from the fact that anisotropic plates have different properties in different directions.

\section{Numerical values of SSSS plate at angle fiber orientation of $\mathbf{1 5}^{\mathbf{0}}$}

Table 4.3 shows that out-plane displacement values (w) and the in-plane displacements values ( $\mathrm{u} \&$ v) decrease as the thickness of the plate decreases. These effects act more at the thick plate zone but becomes very negligible at the thing plate zone. The inplane stresses, $\overline{\sigma_{x x}}, \overline{\sigma_{y y}}$, and $\tau_{x y}$ decrease as the plate decreases in thickness vice versa. Also, it is observed that the decrease is more effective at thick and moderately thick plate section but very negligible at the thin plate section. The values of out-plane stresses $\tau_{x z}$ and $\tau_{y z}$ increase as the plate thickness decreases. These decrease of displacements values and increase and decrease of the stresses values of the plate can be explained from the fact that anisotropic plates have different properties in different directions.

\section{Numerical values of SSSS plate at angle fiber orientation of $30^{\circ}$}

We observed from Table 4.3 that out-plane displacement values (w) and in-plane displacement values ( $\mathrm{u} \& \mathrm{v}$ ) decrease as the thickness of the plate decreases. The decrease was very noticeable at the thick plate zone but gradually diminishes as the thickness decreases. This shows that the displacements are more effective on thick plate than thin plate. The in-plane stresses, $\overline{\sigma_{\mathrm{xx}}}, \overline{\sigma_{\mathrm{yy}}}$ and $\tau_{x y}$ decrease as the plate thickness decreases vice versa. It is observed that the decrease is more noticeable at the thick plate section but gradually decreases at the thin plate section. The values of outplane stresses $\tau_{x z}$ and $\tau_{y z}$ increase as the plate thickness decreases. These decrease of displacements values and increase and decrease of the stresses values of the plate can be explained from the fact that anisotropic plates have different properties in different directions.

\section{Results of numerical values of SSSS plate at angle fiber orientation of $45^{\circ}$}

It is observed from Table $4.2 \mathrm{~d}$ that out-plane displacement values (w) and in-plane displacements values ( $u$ \& v) decrease as the thickness of the plate decreases. The decrease are more effective at the thick plate zone but was gradual at the thin plate zone. The in-plane stresses, $\overline{\sigma_{\mathrm{xx}}}, \overline{\sigma_{\mathrm{yy}}}$, and $\tau_{x y}$ and the out-plane stresses $\tau_{x z}$ and $\tau_{y z}$ increases as the plate thickness decreases vice versa. This increase in stresses as the plate thickness decreases are very obvious at the thick plate section but gradually decreases as the thickness of the plate decrease. At $45^{\circ}$ angle fiber orientation, the out-plane and the in-plane displacements decrease as the plate thickness decreases while both the out-plane 
and the in-plane stresses increase as the plate thickness decreases.

\section{Results of numerical values of SSSS plate at angle fiber orientation of $\mathbf{6 0}^{\circ}$}

Table 4.5 shows that out-plane displacement (w) values and in-plane displacements (v) values decrease as the thickness of the plate decreases. The decrease was much at the thick plate section but was gradual at the thin plate section. This shows that the out-plane displacement are more effective on thick plate than thin plate. The values of the in-plane stresses, $\overline{\sigma_{x x}}$, and $\overline{\sigma_{y y}}$ and the out-plane stresses $\tau_{x z}$ and $\tau_{y z}$ increase as the plate thickness decreases vice versa while the values of the in-plane stress $\tau_{x y}$ decreases as the thickness of the plate decreases. Both the increase and decrease of the displacements and stresses are more effective at the thick plate section but becomes gradual as the thickness of the plate decreases. At $60^{\circ}$ angle fiber orientation both the out-plane and the in-plane displacements decreases as the plate thickness decreases while both the out-plane and the in-plane stresses increases as the plate thickness decreases except the values of the in-plane stress $\tau_{x y}$ which decreases as the plate thickness decreases. These decrease of displacements values and increase and decrease of the stresses values of the plate can be explained from the fact that anisotropic plates have different properties in different directions.

\section{Results of numerical values of SSSS plate at angle fiber orientation of $75^{\circ}$}

We observed from Table 4.6 that out-plane displacement values $(\mathrm{w})$ and in-plane displacements (u $\&$ v) decrease as the thickness of the plate decreases. The decrease was more effective at the thick plate zone than at the thin plate zone. The in-plane stresses, $\overline{\sigma_{x x}}$, and $\tau_{x y}$ decrease in values as the plate thickness decreases while the in-plane stress $\overline{\sigma_{y y}}$ and out-plane stresses $\tau_{x z}$ and $\tau_{y z}$ increase in values as the plate thickness decreases vice versa. These decrease of displacements values and increase and decrease of the stresses values of the plate can be explained from the fact that anisotropic plates have different properties in different directions.

\section{Results of numerical values of SSSS plate at angle fiber orientation of $\mathbf{9 0}^{\circ}$}

Table 4.7 shows that out-plane displacement values $(\mathrm{w})$ and in-plane displacements ( $\mathrm{u} \& \mathrm{v})$ values decrease as the thickness of the plate decreases. The decrease was more at the thick plate section than at the thin plate section. This shows that the out-plane displacements are more active on thick plate than thin plate. The values of the in-plane stresses, $\overline{\sigma_{x x}}, \overline{\sigma_{y y}}$, and $\tau_{x y}$ and the out-plane stress $\tau_{x z}$ decrease as the plate thickness decreases while the values of the out-plane stress $\tau_{y z}$ increases as the thickness of the plate decreases. This increase or decrease in stresses as the plate thickness decreases are very obvious at the thick plate section but gradually decreases as the thickness of the plate decreases. At $90^{\circ}$ angle fiber orientation both the out-plane and the in-plane displacements and stresses values decrease as the plate thickness decreases except the values of the out-plane stress $\tau_{y z}$ which increases as the plate thickness decreases.

\section{Comparison of present study with those from other authors}

The result from present study was compared with the results from SSSS isotropic square plate as obtained by various authors: [7-13]. At, $\alpha=a / t=4$, the percentage difference between present study and [8] for displacement (u) was $-9.52 \%$ which seems mildly under-estimated but gave $-2.59 \%$ when compared with [12]. Also, at $\mathrm{a} / \mathrm{t}=10$, for displacement $(\mathrm{u})$, the percentage differences between present study, [8] and [12] are $-3.73 \%$ and $-2.35 \%$ respectively. For displacements $(\mathrm{w})$ at $\mathrm{a} / \mathrm{t}=4$, the percentage differences between [8] and [12] with present study are $-5.95 \%$ and $-2.61 \%$. At $\mathrm{a} / \mathrm{t}=10$, the percentage differences for [8] and [12] with present study gave $0.64 \%$ and $0.38 \%$ for displacement $(\mathrm{w})$. For in-plane stress $\left(\overline{\sigma_{\mathrm{xx}}}\right)$, at a/t $=4$, comparing present study with [8] and [12] gave $2.94 \%$ and $7.14 \%$ respectively. For in-plane stress $\left(\overline{\sigma_{x x}}\right)$, at a/t $=10$, the percentage differences between present study, [8] and [12] are $6.68 \%$ and $7.33 \%$. For In-plane stress $\left(\overline{\tau_{x y}}\right)$ at $\mathrm{a} / \mathrm{t}=4$, the percentage differences between the present study, [8] and [12] are $-25.6 \%$ and $-9.85 \%$ respectively. Also for In-plane stress $\left(\overline{\tau_{x y}}\right)$ at $a / t=10$, the percentage differences with present study, [8] and [12] are mildly underestimated as $-14.6 \%$ and $-9.55 \%$. The out-plane stress $\left(\overline{\tau_{x z}}\right)$, at a/t $=4$ and $\mathrm{a} / \mathrm{t}=10$, for [8] gave $-65.9 \%$ and $-55.3 \%$ and gave $13.8 \%$ and $-3.77 \%$ when compared with [12]. From Table 4.8, It is observed that similar methods yield closer values than non-similar methods. The table has confirmed the similarity of present study with previous works, even though the similarity were not very high but, it is good enough to validate the present study.

\section{CONCLUSIONS}

The study presents novel formulas for the analysis of thick rectangular anisotropic plates based on refined plate theory and assumptions. Third order shear deformation theory and Ritz energy method was employed for the analysis. The solution derived novel formulas for the out-plane displacement (w), in-plane displacements ( $\mathrm{u} \& \mathrm{v})$, in-plane stresses $\left(\overline{\sigma_{\mathrm{xx}}}, \overline{\sigma_{\mathrm{yy}}}\right.$ and $\left.\tau_{x y}\right)$ and out-plane stresses $\left(\tau_{x z}\right.$ and $\left.\tau_{y z}\right)$ which were used in the analysis of thick anisotropic rectangular plate. The shear deformation function derived by [14] was employed. The formulas was employed to determine the deflection $(\mathrm{u}, \mathrm{v} \& \mathrm{w})$ at the center of the anisotropic rectangular plate and also stresses $\left(\sigma_{\mathrm{x}}, \sigma_{\mathrm{y}}\right.$, $\tau_{\mathrm{xy}}, \tau_{\mathrm{xz}}$ and $\left.\tau_{\mathrm{yz}}\right)$ at the meaningful points on the thick anisotropic rectangular plate for various angles and various span to thickness ratio (alpha, $\alpha$ ) of the two 
boundary conditions; all four edges simply supported (ssss).

From the numerical and compared results obtained, the following conclusion was drawn. The novel formulas developed for prediction of displacements and stresses in the present study can be used to satisfactory analyze thick anisotropic rectangular plate problems of SSSS boundary condition. The method is simple and can be employed to analyze rectangular plates of other boundary conditions.

\section{REFERENCES}

1. Vasiliev, V. V., \& Morozov, E. (2013). Advanced mechanics of composite materials and structural elements (third Edition). Elsevier: London.

2. Yang, W., \& He D. (2018). Bending, free vibration and buckling analyses of anisotropic layered microplates based on a new size dependent model. Journal of Composite Structures, 189(1), 137 147.

https://doi.org/10.1016/j.compstruct.2017.09.057

3. Lisboa, T.D.V., \& Marczak, R.J. (2017). A recursive methodology for the solution of semianalytical rectangular anisotropic thin plates in linear bending. Journal of Applied Mathematical Modelling, $\quad 48(1), \quad 711 \quad$ - $\quad 730$. https://doi.org/10.1016/j.apm.2017.04.020

4. Ghugal,Y.M., \& Shimpi, R.P. (2002). A review of refined shear deformation theories of isotropic and anisotropic laminated plates. Journal of Reinforced Plastics and Composites, 21(1), 775-813. https://doi.org/10.1177/073168402128988481

5. Nelson, R. B., \& Lorch, D. R. (1974). A refined theory for laminated orthotropic plates. Journal of Applied Mechanics, 41(1), 177 - 183. https://doi.org/10.1115/1.3423219

6. Phan, N. D., \& Reddy J. N. (1985). Analysis of laminated composite plates using a higher order shear deformation theory. International Journal of
Numerical Methods in Engineering, 21(4), 2201 2219. https://doi.org/10.1002/nme.1620211207

7. Reddy J. N. (2004). Mechanics of laminated composite plates and shell "theory and analysis" (Second edition). CRC Press: Washington D.C.

8. Sayyad, A. S., \& Ghugal, Y. M. (2012). Bending and free vibration analysis of thick isotropic plates by using exponential shear deformation theory. Journal of Applied and Computational Mechanics, $6(1)$, 65-82. https://www.researchgate.net/publication/26200448 3

9. Ghugal, Y. M., and Sayyad, A. S. (2010). A static flexure of thick isotropic plates using trigonometric shear deformation theory. Journal o Solid $\begin{array}{lllll}\text { Mechanics, } & \text { 2(1), } & 79 & - & 90 .\end{array}$ https://doi.org/wwwjsm.paper.pdf

10. Ghugal, Y. M., \& Pawar, M. D. (2011). Buckling and vibration of plates by hyperbolic shear deformation theory. Journal of Aerospace Engineering and Technology, 1(1-3), 6 24.https://doi.org/10.37591/.v1i1-3.724

11. Kirchhoff, G.R. (1850). Uber das gleichgewichi und die bewegung einer elastishem scheibe. Journal Fuer die Reine und Angewandte Mathematik, 40, 51-88. https://doi.org/10.1515/crit.1850.40.51

12. Mindlin R.D. (1951). Influence of rotary inertia and shear on flexural motions of isotropic elastic plates. Journal of Applied Mechanics, 18, 31 - 38.

13. Pagano, N. (1970). Exact solutions for rectangular bidirectional composites and sandwich plates. Journal of Composite Materials, 4(2), 20-34. https://doi.org/10.1177/02199837000400102

14. Ibearugbulem, O. M. (2012). Application of a direct variational principle in elastic stability of rectangular flat thin plates. Ph.D Thesis submitted to postgraduate school, Federal University of Technology Owerri. 